\title{
ALINHAMENTO ESTRATÉGICO NA CADEIA DE SUPRIMENTOS: UMA ANÁLISE DE EMPRESAS DO SETOR DE BENS DE CAPITAL A PARTIR DE SUAS PRIORIDADES COMPETITIVAS ${ }^{1}$
}

\section{STRATEGIC ALIGNMENT IN SUPPLY CHAIN: AN ANALYSIS OF COMPANIES IN THE CAPITAL GOODS SECTOR FROM ITS COMPETITIVE PRIORITIES}

\author{
Roberto Giro Moori ${ }^{2}$ \\ Mauricio Henrique Benedetti ${ }^{3}$ \\ Odair Oliva Farias ${ }^{4}$
}

\begin{abstract}
RESUMO: Partindo-se da premissa de que o alinhamento estratégico em cadeias de suprimentos se apoia em escolhas entre fornecedores e compradores, este trabalho teve por objetivo analisar o alinhamento estratégico, por meio das prioridades competitivas. Trata-se de um estudo exploratório de caráter descritivo, realizado em duas fases envolvendo empresas do setor de bens de capital. Na primeira fase utilizou-se um questionário construído com uma escala ordinal comparativa e na segunda fase o questionário utilizado foi construído com uma escala de classificação por prioridades não comparativas. Para a análise dos dados utilizou-se estatística descritiva e testes não-paramétricos nas duas fases e escala somada na segunda fase. De uma maneira geral, os resultados encontrados mostram clara orientação das empresas em serem competitivas, todavia, alguns descompassos encontrados em alguns critérios competitivos apontaram para a necessidade de maior aproximação entre as empresas e estabelecimento de um relacionamento mais cooperativo para o alinhamento estratégico. Ao se comparar os dois métodos de coletas de dados utilizados na pesquisa empírica, foram apurados resultados distintos, revelando a necessidade de se ter a preocupação em apontar e considerar o método a ser utilizado para a interpretação do fenômeno estudado.
\end{abstract}

PALAVRAS-CHAVE: Alinhamento estratégico; prioridades competitivas; cadeia de suprimentos.

ABSTRACT: Starting of the premise that the strategic alignment in supply chains leans on in choices between suppliers and buyers, this work had for objective to analyze the strategic alignment, from the competitive priorities. It is an exploratory study of descriptive character, accomplished in two phases involving companies of the sector of capital goods. In the first phase a questionnaire was used built with a comparative ordinal scale and in the second phase the questionnaire used was built with a classification scale by priorities no comparative. For the analysis of the data it was used descriptive statistics and no-parametric tests in the two phases and a summed scale in the second phase. In a general way, the found results show a strong orientation of the companies in be competitive, though, some mismatches found in some competitive criteria indicated for the need of larger approach between the companies and establishment of a more cooperative relationship for the strategic alignment. Comparing the two methods of collections of data used in the empiric research, different results were noted, revealing the need to have the concern in to indicate and to consider the method to be used for the interpretation of the studied phenomenon.

KEY-WORDS: Strategic alignment; competitive priorities; supply chain.

\footnotetext{
${ }^{1}$ Artigo Recebido em 31.01.2011. Revisado por pares em 18.11.2011. Recomendado em 18.11.2011 por Edson Roberto Scharf - Editor Responsável. Publicado em 13.06.2012.

Organização Responsável pelo periódico: Universidade Regional de Blumenau - FURB - www.furb.br/rn
}

\footnotetext{
${ }^{2}$ Universidade Presbiteriana Makenzie- roberto.g.moori@gmail.com

${ }^{3}$ Universidade Presbiteriana Makenzie- mhbenedetti@uol.com.br

${ }^{4}$ Universidade Católica de Santos- UniSantos- odairof@yahoo.com.br
} 


\section{INTRODUÇÃO}

O desenvolvimento de uma estratégia em que se procura obter e manter vantagem competitiva está intimamente ligado com a interação que existe entre os níveis da estrutura da organização. Enquanto a alta administração ocupa-se da estratégia corporativa, a qual envolve aspectos ambientais em que a empresa atua, a elaboração da estratégia de negócios busca a adequação das oportunidades vislumbradas pela estratégia corporativa aos recursos da empresa. Em um nível operacional está a estratégia funcional, orientada a definir e obter os requisitos funcionais demandados pela estratégia de negócios (ou corporativa quando na organização existir apenas uma unidade de negócio) e também definir competências distintivas da empresa.

Em muitos casos, as organizações orientam a formulação de sua estratégia a partir de uma visão limitada à própria empresa, como se sua operação não dependesse de elementos externos e ligados a ela. Este é o caso comum em cadeias onde prevalece a integração vertical e todo o processo, ou grande parte dele, que envolve fornecimento, processamento e distribuição, está por conta de uma única organização. A partir do final do século XX, a dinâmica dos negócios na economia globalizada levou as empresas a repensarem esse modelo de concentração e fechado para poderem reagir com maior rapidez e eficiência às mudanças que o ambiente exigia.

Houve então uma mudança de paradigma em que a vantagem competitiva não se sustentava mais apenas pelas forças da própria empresa, mas pela participação de seus parceiros com os quais estava envolvida e dependia para operar seus negócios. Desta forma, a competitividade nos negócios deixou de basear-se em vantagens competitivas das empresas individualmente para uma competitividade entre cadeias de suprimentos. Nesse sentido, há a necessidade de um alinhamento estratégico dos processos de negócios, tendo como foco a integração interna e externa, ou seja, elaboração de planos e ações que alinhem as operações em nível funcional da empresa com sua estratégia organizacional e também a orientação estratégica das organizações com as quais se relaciona em sua cadeia de suprimentos.

$\mathrm{Na}$ busca pela competitividade por meio de grupos de empresas, em detrimento de empresas individuais, está o cerne da gestão da cadeia de suprimentos. As empresas industriais com estruturas menos verticalizadas e mais focadas nas competências essenciais passam a cooperar junto ao grupo de empresas com as quais se relaciona para obter sucesso individual. Atualmente, setores como o de bens de capital mecânico enfrentam períodos alternados de economia aquecida e recessão global, o que aumenta sobremaneira as exigências em termos de alinhamento entre os elos da cadeia de suprimentos.

Assim, partindo-se da premissa de que o alinhamento estratégico em cadeias de suprimentos se apoia em escolhas entre fornecedores e compradores, levantou-se a seguinte questão para este estudo: as prioridades competitivas das empresas do setor de bens de capital refletem um alinhamento estratégico da cadeia de suprimentos à qual pertencem? $\mathrm{O}$ objetivo central deste artigo foi verificar se as empresas do setor de bens de capital mecânico possuem prioridades competitivas que se alinhem às suas estratégias e às estratégias de seus parceiros ao longo da cadeia de suprimentos. 
O trabalho foi estruturado com a primeira seção apresentando uma revisão da literatura abordando os relacionamentos da cadeia de suprimentos, prioridades competitivas e estratégia de operações. Em seguida os procedimentos metodológicos empregados na pesquisa de campo são apresentados para que o leitor compreenda como os dados empíricos foram coletados e analisados. A seção seguinte do artigo contém as análises dos dados obtidos. Por fim, são apresentadas as conclusões e sugestões que orientam estudos futuros.

\section{REVISÃO DA LITERATURA}

\subsection{Relacionamentos da Cadeia de Suprimentos}

A administração da logística, quando bem realizada, desde o planejamento da rede até o controle de suas atividades, possibilita à organização oferecer diferenciais como de tempo, por uma eficiente gestão de estoques, ou de lugar, por uma eficiente gestão de transporte (CHOPRA; MEINDL, 2003). Sendo assim, por meio da logística é possível uma gestão eficiente do fluxo físico de suprimento de materiais, produtos semi-acabados ou acabados, assim como as informações que são transmitidas por toda a cadeia pela qual circulam os materiais.

Nos dias de hoje, empresas de várias partes do mundo, incluindo as empresas brasileiras, utilizam o potencial da integração das atividades logísticas para aumentarem sua competitividade. O termo "Cadeia de Suprimento" destina-se a designar como um todo a estrutura projetada adequadamente para atender à demanda de um mercado específico (SLACK, 1993). Este conceito pressupõe a adoção coordenada de estratégias apropriadas entre os parceiros de negócios.

Uma visão ampliada dos conceitos de distribuição física e logística, integrando as atividades dessas áreas, fez com que surgisse um novo termo: o Supply Chain Management (SCM). Entra a ideia de coordenação ao longo do canal de distribuição, que inclui as compras, produção e distribuição física. Assim sendo, a administração da logística é uma parte do SCM que planeja, implementa e controla a eficiência do fluxo em ambas as direções do fornecimento de produtos, serviços e informações relacionadas entre o ponto de origem e o ponto de consumo para atender às necessidades dos consumidores (BALLOU, 2006). O SCM integra as atividades logísticas de empresas que trocam entre elas materiais e informações ao longo de um canal de distribuição, ou seja, atividades de outbound para um e inbound para outro. A integração dos processos de negócio a partir do usuário final até os primeiros fornecedores formam o SCM, o que inclui o fornecimento de produtos, serviços e informações, de tal forma que seja adicionado valor ao longo da cadeia a fim de atender necessidades de clientes e demais stakeholders (LAMBERT; COOPER, 2000). Para Croxton et al (2001) o alinhamento dos processos entre as empresas de uma cadeia de suprimentos é de fato a próxima grande fronteira para a redução de custos, aumento da qualidade e da velocidade das operações. Na concepção do SCM, o fluxo dos materiais é puxado, baseado nas necessidades dos consumidores. A manufatura deve ser flexível o suficiente para atender as mudanças do mercado e alianças estratégicas são estabelecidas com um pequeno grupo de fornecedores. 
Ao fazer uma revisão da literatura a respeito do SCM, Tan (2000) observou o predomínio de dois caminhos distintos: (a) compras e suprimentos; e (b) transporte e funções logísticas. Na perspectiva de compras e suprimentos da literatura, o foco está nas funções de compra e gestão de suprimentos de compradores industriais, enquanto na perspectiva de transporte e funções logísticas engloba-se o transporte e as funções de distribuição física de atacadistas e varejistas. A ideia é integrar esses dois caminhos e unificar o SCM em uma comum e aceitável terminologia que envolve todas as atividades de valor ao longo da cadeia.

Quando as duas perspectivas apresentadas por Tan (2000) a respeito do SCM são integradas, aparece a importância de incorporar a gestão da cadeia de suprimentos em todos os processos de planejamento dos negócios. As primeiras abordagens sobre os relacionamentos em cadeias de suprimentos eram essencialmente unidimensionais, menos focadas nos processos de negócio e mais focadas nas escolhas unilaterais entre os elos da cadeia de suprimentos. Estas abordagens unidimensionais incluíam as teorias de custo de transação e da escola de marketing e vendas.

A adoção da gestão da cadeia de suprimentos procura integrar a estratégia ao longo da cadeia para criar processos de manufatura e funções logísticas alinhados por toda a cadeia de suprimentos como uma efetiva arma competitiva que seja difícil de ser superada ou duplicada pela concorrência. Para que este processo possa funcionar corretamente, é necessário um sistema de informações sofisticado e eficaz. Porém, a cadeia precisa ser simples, para que as informações possam trafegar sem restrições que emperrem os processos. Lambert e Cooper (2000) destacam a importância de um eficiente processo de gestão do serviço prestado ao cliente, capaz de prover informações em tempo real a respeito das datas de expedição e disponibilidade de produtos, funcionando como interface entre as organizações de produção e distribuição.

Nos últimos anos a abordagem de gestão de cadeias de suprimentos se desenvolveu aprofundando um pouco mais no relacionamento comprador-fornecedor, pressupondo um relacionamento de confiança, transparência e com relacionamentos colaborativos de longo prazo. As abordagens holísticas e bidimensionais mais recentes propõem uma análise do relacionamento entre fornecedores e compradores entendendo as motivações e objetivos nos dois lados das interfaces.

A perspectiva de poder no relacionamento entre compradores e fornecedores além de holística está explicitamente focada no relacionamento de troca entre os participantes. Para esta escola a relação de poder é o fator determinante dos resultados comerciais e operacionais das transações (MALONI; BENTON, 2000). A associação desta abordagem com o mapeamento do portfólio de relacionamentos contribui para examinar de forma detalhada o alinhamento entre fornecedores e compradores. Segundo Cox et al (2004) o alinhamento efetivo dos objetivos comerciais e operacionais dos compradores e fornecedores elimina o desperdício de tempo e recursos disponíveis nas empresas.

\subsection{Alinhamento Estratégico e Prioridades Competitivas}

A complexidade do relacionamento entre os elos da cadeia de suprimentos, a estrutura econômica dinâmica e a diversidade de opções estratégicas demandam, de fato, uma maior 
racionalidade na pesquisa de setores específicos com uma maior compreensão sobre os relacionamentos nas interfaces das cadeias de suprimentos. $\mathrm{O}$ alinhamento das cadeias de suprimentos depende basicamente dos objetivos estratégicos comerciais dos participantes, dos meios operacionais, estilos de relacionamento, relação de poder exercida entre eles e pela influência dos fatores limitantes e manipuláveis.

À medida que a competição e as incertezas de demanda se intensificam, aumenta a necessidade da empresa focar em prioridades que satisfaçam as necessidades de seus clientes. Todavia, prioridades competitivas em um setor da economia podem não ter a mesma importância para a estratégia de empresas que atuam em outros setores (LAU, 2002). De maneira semelhante, Flynn e Flynn (2004) ressaltam que, além do setor em que a indústria opera, as prioridades competitivas estarão sujeitas a contingências ambientais, o que influenciará significativamente no desenvolvimento e acumulação de capabilidades.

Buscar a superação dos concorrentes pode significar desenvolver competências internas que levam a organização a obter resultados superiores em funções que sejam base para o alcance de seus objetivos estratégicos. Na visão de Slack (2005), a importância da estratégia de operações fica evidente quando associada à estratégia geral da organização, pois contribui para a geração de receita e redução de custos, como por exemplo, em assuntos que têm recebido grande atenção, como TQM e produção enxuta. O desenvolvimento conceitual da estratégia de operações ganha força com teorias como a RBV - visão baseada em recursos (WERNEFELT, 1984; BARNEY, 1995), sendo possível considerar sua influência para o aumento da lucratividade das organizações.

Uma forma eficaz de alinhar a capacidade dos recursos produtivos com as necessidades de mercado é apresentada por Hill (1993), que discute a necessidade de entender os critérios ganhadores e qualificadores de pedidos. Os critérios ganhadores de pedidos são os fatores que obtêm pedidos no mercado, tirando-os da concorrência; os critérios qualificadores de pedidos são os fatores que a empresa precisa alcançar simplesmente para atuar no mercado. Não obstante, Brown et al. (2006) alertam que os critérios ganhadores e qualificadores de pedidos podem mudar com o tempo. Por conta disso, se faz necessário uma avaliação dos critérios ganhadores e qualificadores de pedidos em um processo contínuo e dinâmico.

Para Vokurka, Zank e Lund (2002) as cadeias de suprimentos podem melhorar seu desempenho desenvolvendo prioridades competitivas na sequência especifica de qualidade, confiabilidade, flexibilidade, rapidez e finalmente eficiência em custo, o que deve posicionála da melhor forma frente aos principais desafios competitivos.

Droge, Vickery e Markland (1994), apontam que as prioridades competitivas irão variar de acordo com o tipo de empresa em questão, o ambiente no qual está inserida e ainda pela percepção de seus gestores, enfatizando a importância do alinhamento das prioridades competitivas com uma análise ambiental e a estratégia da organização. Uma pesquisa realizada por esses autores em indústrias de móveis para escritórios e residências, cujo modelo continha 31 prioridades competitivas para três áreas funcionais da empresa (marketing, manufatura e desenvolvimento de produtos), revelou que das dez prioridades competitivas mais importantes, oito eram de responsabilidade das áreas de manufatura ou marketing. 
Em pesquisa realizada com grandes empresas manufatureiras do Japão, EUA e Europa, De Meyer e seus colaboradores observaram que as empresas japonesas priorizam o desenvolvimento de habilidades, capazes de responder às demandas flutuantes e oferecer ampla variedade de produtos com ciclos de vida cada vez mais reduzidos. Para isso, consideram importante serem flexíveis, mas sem perderem a eficiência em produzir com baixos custos. As empresas da Europa estão mais direcionadas em reduzir seus custos produtivos para se tornarem mais competitivas, investindo em novas tecnologias para automação do trabalho e sistemas de controle de estoque. A qualidade é a prioridade para as empresas norte-americanas, onde procuram melhorar seus processos para que ofereçam qualidade mínima, para depois aplicarem a automação (DE MEYER et al., 1989).

O desempenho da empresa se refere aos aspectos observados no âmbito externo (visão do cliente) assim como no âmbito interno (fatores que servirão de base para o desempenho externo). Os aspectos qualidade, velocidade, confiabilidade, flexibilidade e custo são apontados por Slack et al. (2002) como os elementos básicos da competitividade.

O critério qualidade representa vantagem competitiva para as empresas por meio de produtos com alto desempenho, isentos de erros, e com características que os outros competidores não conseguem oferecer (HAYES; PISANO, 1996). Qualidade influenciará outros fatores competitivos, tais como em confiabilidade (pois reduz a probabilidade de faltar o produto e aumenta a estabilidade e a eficiência da organização) e em custos (pois promove menos desperdício de material, menos tempo perdido para correção, menos confusão, menos irritação) (SLACK et al., 2002).

A flexibilidade pode ser subdivida em dois grupos: variedade de produtos e flexibilidade de volume. Normalmente, empresas de pequeno porte irão competir na flexibilidade de variedade de produtos, pois significa dizer que elas podem atender pedidos fora dos padrões e que podem tomar a frente na introdução de novos produtos (HAYES; PISANO, 1996). Flexibilidade terá um efeito em outros fatores competitivos, tais como velocidade, custos, e confiabilidade, visto que operações flexíveis adaptam-se rapidamente às circunstâncias mutantes e não interrompem a produção (SLACK et al., 2002).

A velocidade refere-se à diminuição no tempo de entrega de um determinado produto ou serviço solicitado pelo cliente externo. Para alcançar este fator competitivo é preciso diminuir o tempo no processo da manufatura, o que irá beneficiar a empresa e sua operação deixando-a mais enxuta e mais produtiva (HAYES; PISANO, 1996). A diminuição do tempo no processo de manufatura trará também uma maior satisfação do consumidor, maior confiabilidade e consequentemente redução dos custos na manufatura (SLACK et al., 2002).

A confiabilidade é atingida ao se cumprir os prazos de entrega de produto ou serviço ao consumidor final. Está diretamente ligada à velocidade de entrega, que significa fazer as coisas em tempo para que esses recebam os bens ou serviços honrados em contrato (HAYES; PISANO, 1996). Os consumidores só podem julgar a confiabilidade de uma operação após o produto ou serviço ter sido entregue. Ao selecionar o serviço pela primeira vez, o cliente não terá qualquer referência do passado quanto à confiabilidade do fornecedor, entretanto no decorrer do tempo, a confiabilidade poderá assumir a posição de fator mais importante do que qualquer outro critério em um vínculo de fornecimento (CHASE, et al, 2001). 
O custo é normalmente um dos principais objetivos da produção (HAYES; PISANO, 1996). O foco da gestão de operações, como um todo, é na redução dos custos totais de produção (SLACK et al, 2002). Mesmo as empresas que concorrem no mercado, utilizando outros objetivos de desempenho para atrair seus clientes terão interesse em manter seus custos baixos. Tentar obter uma vantagem de custo não significa que a empresa estará oferecendo produtos de baixo valor ou baixa qualidade (HEIZER; RENDER, 2001).

Esses cinco elementos, entretanto, levando-se em consideração os fatores internos de manufatura, devem trabalhar de forma integrada e concisa na busca do objetivo alvo (competitividade). Todos os fatores citados estão intimamente relacionados entre si, formando uma cadeia onde não será possível alcançar um fator, sem a existência dos outros fatores atuando de forma direta ou indireta (HAYES; PISANO, 1996; SLACK et al, 2002).

\section{PROCEDIMENTOS METODOLÓGICOS}

Este artigo apresenta uma análise do alinhamento estratégico das empresas do setor de bens de capital, a qual pode ser classificada como resultado de uma pesquisa de natureza exploratória do tipo descritivo. Para a realização da referida análise, um modelo orientador foi utilizado, como mostrado na Figura 1.

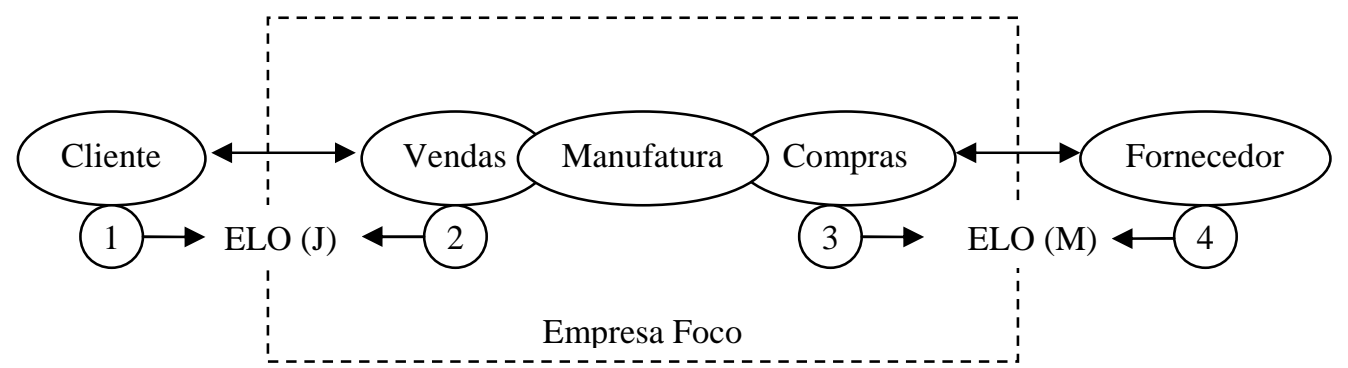

Figura 1: Modelo orientador da pesquisa

Fonte: Os autores

Observa-se pela Figura 1, os elos a jusante e a montante da empresa foco representados pelos ELO (J) e ELO (M), respectivamente, sendo que: a) os números (1) e (4) representam as empresas first tier ou elos imediatos, em uma relação dyadic; b) o número (2) representa a área de vendas da empresa foco e interage com o cliente (1) e; c) o número (3) representa a área de compras e interage com o fornecedor (4). Com base no modelo apresentado na Figura 1, a operacionalização da análise se deu em duas fases. Apesar da coleta de dados ter ocorrido em dois momentos (fases) não se trata de um estudo longitudinal, uma vez que em cada fase aplicou-se escalas diferentes para as respostas e os questionários foram aplicados a amostras diferentes. A realização da coleta de dados em dois momentos utilizando métodos diferentes teve como objetivo verificar se o método escolhido interfere nos resultados a ponto de gerar diferentes interpretações a respeito do mesmo fenômeno. Em ambas as fases, os dados foram tratados por meio do pacote de software estatístico SPSS Statistical Package for the Social Sciences. 


\section{ALINHAMENTO ESTRATÉGICO NA CADEIA DE SUPRIMENTOS: UMA ANÁLISE DE EMPRESAS DO SETOR DE BENS DE CAPITAL A PARTIR DE SUAS PRIORIDADES COMPETITIVAS}

Primeira Fase: A realização desta primeira fase ocorreu no período de fevereiro de 2007 a janeiro de 2008. A coleta de dados foi realizada por meio de um questionário em que os respondentes eram solicitados a classificar em ordem de importância quatro prioridades competitivas dentre dez apresentadas. Os questionários foram enviados aos respondentes por e-mail e, em alguns casos, entregues pessoalmente nas empresas. Os dados coletados foram segmentados em três blocos. O primeiro bloco referiu-se ao nome do respondente, função, tempo na empresa e formação acadêmica. $\mathrm{O}$ segundo bloco referiu-se à empresa, contendo informações como o nome e ramo de atividade da empresa, tipo de produção, número de empregados e faturamento. No terceiro bloco buscou-se saber como os respondentes se posicionavam em relação aos critérios ganhadores, qualificadores e pouco importantes na hora da compra. Para cada critério foi solicitado ao respondente, de um total de 10 prioridades competitivas (ou critérios ganhadores de pedidos) apresentados em uma lista, que ordenasse os 4 primeiros, que na sua percepção eram os mais significativos para sua empresa. Para delimitar as variáveis, realizou-se levantamento bibliográfico sobre os critérios ganhadores, qualificadores e pouco importantes mais significativos e, de preferência, que já tivessem sido aplicados em empresas do setor de bens de capital. A lista de critérios ganhadores de pedidos, relacionados à decisão de natureza administrativa e de engenharia, é mostrada na Tabela 1.

Tabela 1: Critérios Ganhadores de Pedidos

\begin{tabular}{|c|c|c|c|}
\hline \multicolumn{2}{|c|}{ CÓDIGO } & \multirow{2}{*}{\begin{tabular}{|l|} 
Critérios Ganhadores de Pedidos \\
Flexibilidade de data de entrega
\end{tabular}} & \multirow{2}{*}{\begin{tabular}{|l} 
DESCRIÇÃO \\
Capacidade da empresa em modificar os prazos de \\
entrega para se adaptar a uma mudança de cronograma
\end{tabular}} \\
\hline \multirow{5}{*}{ 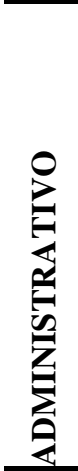 } & A1 & & \\
\hline & A2 & $\begin{array}{l}\text { Preço (equipamento } \\
\text { componente) }\end{array}$ & $\begin{array}{l}\text { Capacidade de o fornecedor oferecer equipamento de } \\
\text { preço baixo comparado com similares da concorrência }\end{array}$ \\
\hline & A3 & Velocidade da data de entrega & $\begin{array}{l}\text { Capacidade do fornecedor de fabricar em prazo mais } \\
\text { curto que os concorrentes }\end{array}$ \\
\hline & A4 & Confiabilidade da data de entrega & $\begin{array}{l}\text { Capacidade da empresa em entregar o equipamento } \\
\text { adquirido dentro do prazo estipulado inicialmente }\end{array}$ \\
\hline & A5 & $\begin{array}{l}\begin{array}{l}\text { Flexibilidade de alteração } \\
\text { contratual }\end{array} \\
\end{array}$ & $\begin{array}{l}\text { Capacidade da empresa de aceitar modificações no } \\
\text { contrato durante o período de fabricação do equipamento }\end{array}$ \\
\hline \multirow{6}{*}{ 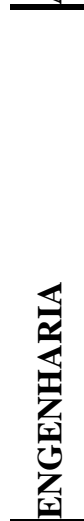 } & E1 & Confiabilidade às normas & $\begin{array}{l}\text { Capacidade do fornecedor de atender às especificações } \\
\text { das normas internas de segurança em meio ambiente }\end{array}$ \\
\hline & $\mathrm{E} 2$ & Confiabilidade ao projeto & $\begin{array}{l}\text { Capacidade do fornecedor de construir um equipamento } \\
\text { que esteja em conformidade com o projeto original }\end{array}$ \\
\hline & E3 & Desempenho do equipamento & $\begin{array}{l}\text { Capacidade do equipamento de desempenhar as funções } \\
\text { para as quais foi projetado }\end{array}$ \\
\hline & $\mathrm{E} 4$ & Confiabilidade do equipamento & $\begin{array}{l}\text { Capacidade do equipamento em operar sem interrupção } \\
\text { no maior tempo possível, antes de ocorrer a falha }\end{array}$ \\
\hline & E5 & Flexibilidade de alteração técnica & $\begin{array}{l}\text { Capacidade da empresa de aceitar modificações técnicas } \\
\text { no equipamento durante o período de fabricação }\end{array}$ \\
\hline & 11 & Outros... & Outros... \\
\hline
\end{tabular}

Fonte: Adaptado de Paiva et al. (2004)

As empresas foram selecionadas por acessibilidade e conveniência, considerando-se como critério para a entrega dos questionários a necessidade do respondente ser gestor dentro da cadeia de suprimentos, como os gerentes de compras, vendas, produção e logística. 
Portanto, utilizou-se uma amostra não-probabilística com a orientação de obter maior homogeneidade e baixa variância, optando-se pelo segmento de fabricantes de máquinas e equipamentos do setor de bens de capital.

Para tratamento dos dados coletados utilizou-se a estatística descritiva, em especial, a frequência de respostas. Utilizou-se ainda, a estatística inferencial pela aplicação de testes não paramétricos, para comparar as populações das empresas foco, clientes e fornecedores relacionados às variáveis de prioridades competitivas e, assim, verificar o alinhamento das prioridades competitivas entre as populações.

Vale ressaltar que como se utilizou uma escala ordinal do tipo comparativo, os dados indicavam a posição relativa, mas não a magnitude das diferenças entre as prioridades. Uma prioridade competitiva classificada em primeiro lugar apresenta características em maior grau do que a prioridade classificada em segundo lugar, mas não se sabe se a prioridade classificada como primeiro está próxima ou afastada da classificada em segundo lugar.

O passo seguinte da pesquisa foi verificar o alinhamento estratégico das prioridades competitivas, por meio da aplicação de técnicas da estatística não-paramétrica: i) teste de Mann-Whitney para comprovar se duas amostras independentes foram ou não extraídas da mesma população; ii) teste de Kolmogorov-Smirnov para comparar se as duas amostras foram extraídas da mesma população (ou de populações com mesma distribuição) e; iii) Quiquadrado $\left(\chi^{2}\right)$ para analisar a associação ou dependência, entre duas variáveis de dois grupos de amostras.

Segunda Fase: Esta etapa da pesquisa ocorreu no período de fevereiro e março de 2009. A coleta de dados se deu por meio de questionário estruturado da mesma forma que na fase anterior, exceto por utilizar no bloco três a escala de classificação por prioridades não comparativa. As prioridades corresponderam à descrição dos critérios ganhadores de pedidos, mostrada na Tabela 1. Assim, foi solicitado ao respondente que assinalasse com um ' $x$ ', na prioridade o respectivo grau de concordância, que variava entre Discordo Totalmente (DT = 1) a Concordo Totalmente $(\mathrm{CT}=6)$. Salienta-se que nesta escala, o respondente não compara a assertiva que está sendo classificada, seja com outra assertiva ou com algum padrão especificado (ou benchmarking). Além disso, ele avalia apenas uma assertiva por vez na categoria de resposta que melhor descreve a prioridade que está sendo classificada. Definido o questionário escolheu-se uma amostra de empresas a partir do cadastro de contato da empresa de consultoria IMAM e contatos pessoais com as empresas do setor de bens de capital.

O tratamento dos dados coletados por meio da escala de classificação por prioridade não comparativa foi realizado utilizando: a) Estatística descritiva, onde foram analisadas as três principais propriedades que descrevem um conjunto de dados numéricos: tendência central (média e mediana), variação (coeficiente de variação) e formato (simetria e achatamento); b) Escala somada, com a finalidade de comparar o grau de concordância entre dois grupos de mesma prioridade coletadas, na primeira e segunda fase, que se deu por métodos diferentes. Na escala somada, as pontuações de cada prioridade foram somadas para produzir uma pontuação final para a prioridade e representada pela média ponderada (Mda). Além disso, a escala somada foi utilizada para analisar o grau de concordância em relação a uma série de prioridades relacionadas a um fator comum e representada pela média geral 


\section{ALINHAMENTO ESTRATÉGICO NA CADEIA DE SUPRIMENTOS: UMA ANÁLISE DE EMPRESAS DO SETOR DE BENS DE CAPITAL A PARTIR DE SUAS PRIORIDADES COMPETITIVAS}

ponderada. Salienta-se que, um indicador é considerado de boa qualidade quando todas as medidas das prioridades têm uma relação coerente entre si no esforço de medir o fator comum em consideração. Para tanto, utilizou-se o coeficiente Alfa $(\alpha)$ de Chronbach como um medidor eficiente para a representação do fator comum (HAIR et al., 2005).

\section{ANÁLISE DOS DADOS E RESULTADOS}

\subsection{Primeira Fase: Coleta de Dados pela Ordenação de Prioridades Competitivas a) Perfil dos Respondentes e das Empresas}

Os dados obtidos foram referentes a 59 respondentes, tendo como principais características: $78 \%$ dos respondentes possuíam cursos superiores em administração e engenharia, ocupando diferentes funções como Compras, Logística, Qualidade e Produção, dos quais 53\% eram supervisores, gerentes e diretores, com tempo de ocupação superior a 5 anos. De forma geral, o perfil dos respondentes foi caracterizado por mão de obra qualificada e com baixo nível de rotatividade. Em relação às empresas, a amostra apresentou uma concentração de empresas na Grande São Paulo com 51\% do total das respostas. Quanto ao ramo de atividade, as empresas pertencentes ao setor metal mecânico foram as que se destacaram com $54 \%$ da amostra. Com relação ao porte, segundo a classificação do BNDES (2008) e SEBRAE (2008), 53\% da amostra eram de empresas de grande porte.

\section{b) Alinhamento Estratégico a Jusante}

A análise dos dados coletados e os resultados sobre o alinhamento estratégico entre as empresas da cadeia de suprimentos, tratados pelas técnicas da estatística descritiva e da indutiva não-paramétrica, são mostrados adiante, na Tabela 2.

Tabela 2: Porcentagem (\%) de Frequência de Resposta (Fr) a Jusante

\begin{tabular}{|c|c|c|c|c|c|c|c|c|c|c|}
\hline \multirow{3}{*}{\multicolumn{2}{|c|}{ RITÉRIOS GANHADORES DE PEDIDOS }} & \multirow{3}{*}{$\%$} & \multicolumn{4}{|c|}{ JUSANTE } & \multicolumn{4}{|c|}{ EMPRESA FOCO } \\
\hline & & & \multicolumn{4}{|c|}{ Cliente (1) } & \multicolumn{4}{|c|}{ Vendas (2) } \\
\hline & & & $1^{\circ}$ & $2^{\circ}$ & $3^{\circ}$ & $4^{\circ}$ & $1^{\mathrm{o}}$ & $2^{\circ}$ & $3^{\circ}$ & $4^{\circ}$ \\
\hline A1 & Flexibilidade de data de entrega & $\mathrm{Fr}$ & 14 & 8 & 8 & 6 & 0 & 0 & 10 & 0 \\
\hline $\mathrm{A} 2$ & Preço (equipamento ou componente) & Fr & 37 & 24 & 16 & 8 & 50 & 10 & 0 & 25 \\
\hline A3 & Velocidade da data de entrega & $\mathrm{Fr}$ & 2 & 12 & 4 & 18 & 10 & 10 & 20 & 0 \\
\hline A4 & Confiabilidade da data de entrega & Fr & 8 & 12 & 24 & 10 & 0 & 10 & 0 & 0 \\
\hline A5 & Flexibilidade de alteração contratual & Fr & 0 & 2 & 2 & 8 & 0 & 0 & 0 & 0 \\
\hline E1 & Confiabilidade às normas & $\mathrm{Fr}$ & 2 & 0 & 8 & 12 & 0 & 0 & 0 & 13 \\
\hline E2 & Confiabilidade ao projeto & Fr & 4 & 10 & 8 & 4 & 20 & 0 & 0 & 0 \\
\hline E3 & Desempenho do equipamento & Fr & 22 & 16 & 8 & 8 & 0 & 40 & 10 & 13 \\
\hline E4 & Confiabilidade do equipamento & Fr & 10 & 14 & 12 & 8 & 20 & 0 & 10 & 38 \\
\hline E5 & Flexibilidade de alteração técnica & Fr & 2 & 4 & 4 & 12 & 0 & 20 & 30 & 0 \\
\hline 11 & Outros... & $\mathrm{Fr}$ & 0 & 0 & 6 & 4 & 0 & 10 & 20 & 13 \\
\hline \multicolumn{2}{|c|}{ Frequência Acumulada Total (\%) } & $\mathbf{F r}$ & 100 & 100 & 100 & 100 & 100 & 100 & 100 & 100 \\
\hline
\end{tabular}


Pela Tabela 2 é possível observar que as prioridades competitivas, representadas pelos critérios ganhadores de pedido, da empresa foco não estão totalmente alinhadas ao que seus clientes consideram prioridade no momento da compra. Apesar de tanto fabricante quanto clientes terem posição semelhante em relação ao critério A2 (preço), ou seja, é considerado o mais importante dos dez critérios listados, outros critérios competitivos são percebidos distintamente pelos respondentes da amostra pesquisada. Para o fabricante, depois de preço os critérios competitivos que foram destacados como mais importantes foram a confiabilidade ao projeto (E2) e a confiabilidade do equipamento (E4). Este resultado mostra que, além da empresa fabricante preocupar-se em ter um preço competitivo para ganhar a preferência de seus clientes, ela também está interessada com sua imagem, orientada a fornecer produtos confiáveis, desde o momento do projeto até sua utilização. Por outro lado, porém não de forma contraditória, o critério considerado mais importante pelo cliente, após preço, foi o desempenho do equipamento (E3). Por se tratar de bens de capital, esta constatação é relevante, uma vez que o produto do fabricante em funcionamento deve ter influência direta no processo do cliente. Conforme apontado por Droge, Vickery e Markland (1994), Lau (2002) e Flynn e Flynn (2004), a definição de critérios competitivos deve ser feita considerando-se o setor em que a indústria atua. Sugere-se que os clientes tenham certo receio na aquisição de equipamentos ou componentes de fabricantes com os quais ainda não tenham estabelecido uma relação de confiança, ou seja, algo que pode ocorrer em uma primeira compra, o que se alinha ao que foi colocado por Hayes e Pisano (1996) a respeito da confiabilidade na entrega. Reforça-se então a necessidade do relacionamento mais próximo e forte entre os elos da cadeia de suprimentos ressaltado pela literatura, visto que o cliente espera que a imagem de confiança que o fabricante veicula se traduza em um equipamento de bom desempenho. Ainda reforçando a ideia de falta de alinhamento das prioridades competitivas neste elo da cadeia, observou-se que, enquanto os fabricantes apontaram apenas quatro critérios como sendo de maior importância, para os clientes, dos dez critérios apresentados, apenas a flexibilidade de alteração (A5) não foi considerada como a principal prioridade competitiva.

Há um elemento presente na Tabela 2 que merece reflexão e que pode indicar necessidade de maior atenção do fabricante com relação à sua capacidade de alterar seu prazo de entrega (SLACK et al., 2002). Isto pode ser notado pelo fato do critério A1 (flexibilidade de data de entrega) ter sido o terceiro critério apontado pelos clientes como o mais importante, enquanto esse item foi praticamente ignorado pelos fabricantes.

\section{c) Alinhamento Estratégico a Montante}

Ao considerar a cadeia a montante do fabricante, é possível observar na Tabela 3 certo descompasso nas prioridades competitivas da empresa foco e seus fornecedores. 
ALINHAMENTO ESTRATÉGICO NA CADEIA DE SUPRIMENTOS: UMA ANÁLISE DE EMPRESAS DO SETOR DE BENS DE CAPITAL A PARTIR DE SUAS PRIORIDADES COMPETITIVAS

\begin{tabular}{|c|c|c|c|c|c|c|c|c|c|c|}
\hline \multirow{3}{*}{\multicolumn{2}{|c|}{ GANHADORES DE }} & \multirow{3}{*}{$\%$} & \multirow{2}{*}{\multicolumn{4}{|c|}{\begin{tabular}{|l|} 
EMPRESA FOCO \\
Compras (3) \\
\end{tabular}}} & \multirow{2}{*}{\multicolumn{4}{|c|}{\begin{tabular}{|l|} 
MONTANTE \\
Fornecedor (4) \\
\end{tabular}}} \\
\hline & & & & & & & & & & \\
\hline & & & $1^{\circ}$ & $2^{\circ}$ & $3^{\circ}$ & $4^{\circ}$ & $1^{\circ}$ & $2^{\circ}$ & $3^{\circ}$ & $4^{\circ}$ \\
\hline A1 & Flexibilidade de data de entrega & $\mathrm{Fr}$ & 10 & 30 & 10 & 0 & 12 & 12 & 5 & 8 \\
\hline A2 & Preço (equipamento ou componente) & $\mathrm{Fr}$ & 10 & 0 & 20 & 40 & 24 & 21 & 17 & 18 \\
\hline A3 & Velocidade da data de entrega & Fr & 40 & 10 & 10 & 0 & 19 & 10 & 24 & 15 \\
\hline A4 & Confiabilidade da data de entrega & Fr & 10 & 20 & 10 & 10 & 2 & 12 & 19 & 15 \\
\hline A5 & Flexibilidade de alteração contratual & $\mathrm{Fr}$ & 0 & 10 & 0 & 10 & 0 & 0 & 5 & 3 \\
\hline $\bar{E} 1$ & Confiabilidade às normas & $\mathrm{Fr}$ & 10 & 0 & 0 & 10 & 5 & 7 & 5 & 10 \\
\hline E2 & Confiabilidade ao projeto & $\mathrm{Fr}$ & 20 & 10 & 10 & 0 & 5 & 14 & 7 & 0 \\
\hline E3 & Desempenho do equipamento & $\mathrm{Fr}$ & 0 & 10 & 40 & 10 & 12 & 12 & 5 & 10 \\
\hline E4 & Confiabilidade do equipamento & $\mathrm{Fr}$ & 0 & 10 & 0 & 10 & 17 & 12 & 10 & 0 \\
\hline E5 & Flexibilidade de alteração técnica & $\mathrm{Fr}$ & 0 & 0 & 0 & 10 & 5 & 0 & 5 & 13 \\
\hline 11 & Outros... & $\mathrm{Fr}$ & 0 & 0 & 0 & 0 & 0 & 0 & 0 & 8 \\
\hline Fre & quência Acumulada Total (\%) & $\mathbf{F r}$ & 100 & 100 & 100 & 100 & 100 & 100 & 100 & 100 \\
\hline
\end{tabular}

Enquanto houve uma maior concentração de fabricantes, agora na posição de clientes, que apontaram o critério A3 (velocidade da data de entrega) como o mais importante em sua decisão de compra, o preço do seu produto (A2) foi considerado com maior frequência pelos fornecedores como o mais importante para ter a preferência de seu comprador. Um atenuante do desalinhamento percebido nesse elo é o fato da velocidade de entrega receber a segunda maior frequência de critério mais importante apontado pelo fornecedor. Todavia, esse desencontro de prioridades é ressaltado ao se verificar que o preço foi o critério que apresentou maior frequência (40\%) de opiniões dos respondentes do fabricante, como sendo o quarto critério em importância. Uma vez que o questionário solicitava para que se assinalasse a ordem de importância dos critérios do $1^{\circ}$ ao $4^{\circ}$, nesse caso, preço é o critério menos relevante para o fabricante em sua decisão de aquisição de equipamentos e componentes. Sugere-se que o fornecedor está sujeito a uma forte concorrência em preço e não está disposto a arriscar-se em cobrar um valor adicional que garanta a velocidade de entrega. Uma situação que poderia levar à diminuição da competitividade da cadeia, como apontado por Tan (2002). Por outro lado, o fabricante não se mostra disposto a procurar fornecedores que pratiquem menores preços, mas que não lhe garantam entregar seus pedidos com rapidez. Conforme destacado por Croxton et al (2001), o alinhamento dos processos entre as empresas de uma cadeia de suprimentos tem grande contribuição para que haja melhor qualidade, operações mais rápidas e custos reduzidos.

\subsection{Segunda Fase: Coleta de Dados pela Escala de Concordância a) Perfil dos Respondentes e das Empresas}

A amostra de empresas foi representada por 61 respondentes, os quais eram colaboradores de 50 empresas. As principais características dos respondentes e empresas da amostra foram: a) com relação aos respondentes, $52 \%$ do total possuíam cursos superiores em administração e engenharia, ocupando diferentes funções como Compras, Logística, 
Qualidade e Produção. Os cargos básicos ocupados por estes profissionais, 32\% deles eram supervisores, gerentes e diretores, com tempo de ocupação superior a 5 anos.

De forma geral, o perfil dos respondentes foi caracterizado por mão de obra qualificada e com baixo nível de rotatividade; b) com relação às empresas, $28 \%$ do total estavam sediadas na Grande São Paulo. Quanto ao ramo de atividade, as empresas e respondentes pertencentes ao setor metal mecânico foram os mais representativos da amostra com $82 \%$ das repostas. O porte das empresas, segundo a classificação do BNDES (2008) e SEBRAE (2008), 44\% das empresas, correspondeu a de tamanho grande e o tipo de produção foi equilibrado, sendo $34 \%$ seriada, $36 \%$ por lote e $30 \%$ sob pedido.

\section{b) Alinhamento Estratégico: elo a Jusante}

Os resultados sobre o alinhamento estratégico, a jusante, da empresa foco são mostrados na Tabela 4. Pelos dados apresentados, é possível verificar que os clientes têm como prioridade a confiabilidade na entrega (A4) e o desempenho do equipamento (E3). Nota-se que tais prioridades assemelham-se às prioridades dos fabricantes, tendo em vista que o critério confiabilidade na entrega ficou com a maior média da escala somada ( $M d a=5,5)$, ou seja, o mesmo valor obtido pelas respostas dos clientes, e Mda igual a 5,3 para desempenho do equipamento, próximo de 5,5 dos clientes. Todavia, os fabricantes mostraram-se mais inclinados a concordarem com a importância dada à confiabilidade do projeto e às normas. Revelou-se então uma orientação dos fabricantes em serem percebidos como confiáveis, tanto no que se refere às especificações que atendam às necessidades dos clientes quanto aos prazos de entrega. $\mathrm{O}$ atendimento das necessidades dos clientes e demais stakeholders é um objetivo destacado por Lambert e Cooper (2000) na integração da cadeia de suprimentos, o que significa agregar valor ao longo da cadeia por meio do fornecimento de produtos, serviços e informações. Assim, acredita-se que o fabricante sinta-se seguro quanto ao desempenho de seu produto, mas preocupa-se em fazer com que os clientes tenham essa percepção mesmo antes de receberem seu pedido.

Tabela 4: Elo a Jusante

\begin{tabular}{|c|c|c|c|c|c|c|c|c|c|c|c|c|}
\hline \multirow{2}{*}{ PRIORIDADES COMPETITIVAS } & \multicolumn{6}{|c|}{ CLIENTE (1) } & \multicolumn{6}{|c|}{ Empresa Foco - VENDAS (2) } \\
\hline & Mda & Mdn & $\mathrm{CV}$ & $\mathrm{Sk}$ & $\mathrm{Ku}$ & Rk & Mda & Mdn & $\mathrm{CV}$ & $\mathrm{Sk}$ & $\mathrm{Ku}$ & Rk \\
\hline Flexibilidade de data de entrega & 3,7 & 4,0 & 49,0 & $-0,21$ & $-1,48$ & & 4,8 & 5,0 & 27,1 & $-0,98$ & 0,37 & \\
\hline A2 Preço (eqpto ou componentes) & 4,3 & 4,5 & 34,1 & $-0,59$ & $-0,50$ & & 4,6 & 5,0 & 11,2 & $-0,39$ & $-2,26$ & \\
\hline Velocidade da data de entrega & 4,3 & 4,0 & 32,2 & 0,07 & $-1,34$ & & 5,0 & 5,0 & 22,6 & $-1,82$ & 4,33 & \\
\hline Confiabilidade da data de entrega & 5,5 & 6,0 & 14,8 & $-1,70$ & 2,52 & 2 & 5,5 & 6,0 & 16,5 & $-2,21$ & 5,32 & 2 \\
\hline A5 Flexibilidade de alt. contratual & 2,9 & 3,0 & 47,4 & 0,53 & $-0,28$ & & 4,4 & 4,5 & 20,4 & $-0,15$ & $-0,43$ & \\
\hline E1 Confiabilidade às normas & 5,3 & 6,0 & 17,4 & $-0,93$ & $-0,29$ & 3 & $\overline{5,4}$ & 6,0 & 14,6 & $-0,99$ & $-0,46$ & 3 \\
\hline Confiabilidade ao projeto & 5,3 & 6,0 & 19,8 & $-1,74$ & 3,09 & 4 & 5,5 & 6,0 & 12,3 & $-1,07$ & 0,35 & 1 \\
\hline Desempenho do equipamento & 5,5 & 6,0 & 13,7 & $-1,91$ & 3,97 & 1 & 5,3 & 6,0 & 23,1 & $-2,17$ & 4,77 & \\
\hline Confiabilidade do equipamento & 5,1 & 5,0 & 20,8 & $-1,05$ & 1,02 & & 5,3 & 5,5 & 16,7 & $-1,73$ & 3,81 & 4 \\
\hline Flexibilidade de alteração técnica & 3,3 & 3,0 & 41,1 & 0,22 & 0,01 & & 4,6 & 5,0 & 21,7 & $-1,60$ & 3,83 & \\
\hline Média geral e $\alpha$-Cronback & 4,5 & \multicolumn{2}{|c|}{$\alpha_{\mathrm{T}}=0,604$} & \multicolumn{3}{|c|}{$\alpha_{4}=0,754$} & 5,0 & \multicolumn{2}{|c|}{$\alpha_{\mathrm{T}}=0,867$} & \multicolumn{3}{|c|}{$\alpha_{4}=0,896$} \\
\hline
\end{tabular}

Fonte: Dados da pesquisa

De maneira semelhante, ao se analisar as menores médias, ou seja, os menores níveis de concordância para a importância dos critérios apresentados, verifica-se que os dois critérios 


\section{ALINHAMENTO ESTRATÉGICO NA CADEIA DE SUPRIMENTOS: UMA ANÁLISE DE EMPRESAS DO SETOR DE BENS DE CAPITAL A PARTIR DE SUAS PRIORIDADES COMPETITIVAS}

considerados menos importantes são a flexibilidade de alteração contratual (A5) e flexibilidade de alteração técnica (E5), tanto para clientes quanto para fabricantes, apesar das menores médias obtidas nos dois critérios para os clientes.

Tanto para clientes quanto para a empresa foco, os valores obtidos de Sk (simetria) e $\mathrm{Ku}$ (achatamento), denotaram que se trata de uma distribuição de dados não normal. No geral, as empresas foco, sob a perspectiva da área de vendas (2) apresentou menores limites de coeficiente de variação, maior pontuação na escalada somada, denotando melhor correlação e consistência interna ( $\alpha$-Cronbach) entre as prioridades analisadas, incluindo as primeiras quatro prioridades competitivas.

\section{c) Alinhamento Estratégico: elo a Montante}

Os resultados sobre o alinhamento estratégico, a montante, da empresa foco são mostrados na Tabela 5. A análise do elo a montante a partir dos dados apresentados revelou uma situação diferente da observada no elo a jusante. O maior valor de Mda $(5,7)$ para a empresa foco foi obtido para o critério E4 (confiabilidade do equipamento), enquanto esse mesmo critério recebeu um valor de Mda igual a 4,3 para o fornecedor (sétima posição em relação aos dez critérios). Por outro lado, a principal prioridade apontada pelo fornecedor foi a segunda prioridade para a empresa foco, que é o desempenho do equipamento (E3), com o mesmo valor de Mda $(5,6)$ tanto para fornecedor quanto para fabricante. No outro extremo das prioridades, os três últimos critérios foram os mesmos para ambos (A2, A5 e E5), apenas com alteração da posição entre estas prioridades. Dessa maneira é possível dizer que nem preço, nem flexibilidade de alteração contratual ou técnica são pontos considerados por esse elo da cadeia de suprimentos como importantes para a venda e aquisição de equipamentos ou componentes. Assim sendo, há mais elementos que sugerem alinhamento entre as prioridades competitivas entre fornecedor e fabricante do que o contrário. Observam-se tanto objetivos comerciais quanto operacionais comuns nesse elo, o que pode levar à redução do desperdício de tempo e utilização de recursos como mencionado por Cox et al. (2004).

Tabela 5: Elo a Montante

\begin{tabular}{|c|c|c|c|c|c|c|c|c|c|c|c|c|}
\hline \multirow{2}{*}{ PRIORIDADES COMPETITIVAS } & \multicolumn{6}{|c|}{ Empresa Foco - COMPRAS (3) } & \multicolumn{6}{|c|}{ FORNECEDOR (4) } \\
\hline & Mda & Mdn & $\mathrm{CV}$ & $\mathrm{Sk}$ & $\mathrm{Ku}$ & Rk & Mda & Mdn & $\mathrm{CV}$ & Sk & $\mathrm{Ku}$ & $\mathrm{Rk}$ \\
\hline Flexibilidade de data de entrega & 5,1 & 5,0 & 19,6 & $-0,85$ & $-0,14$ & & 4,4 & 5,0 & 29,5 & $-0,49$ & $-0,61$ & \\
\hline Preço (eqpto ou componentes) & 3,9 & 3,5 & 27,7 & 0,71 & $-0,92$ & & 3,1 & 3,0 & 33,8 & 2,36 & 7,30 & \\
\hline Velocidade da data de entrega & 4,6 & 5,0 & 23,7 & $-1,03$ & 2,05 & & 4,6 & 4,0 & 26,0 & 0,03 & $-1,67$ & 4 \\
\hline Confiabilidade da data de entrega & 5,3 & 5,5 & 24,6 & $-1,32$ & 1,41 & & 4,9 & 5,0 & 16,9 & 0,19 & $-1,49$ & 2 \\
\hline Flexibilidade de alter. contratual & 3,9 & 4,0 & 25,4 & 0,85 & $-0,01$ & & 4,2 & 4,0 & 25,8 & 0,74 & $-0,35$ & \\
\hline E1 Confiabilidade às normas & 5,4 & 5,5 & 12,3 & $-0,74$ & $-0,19$ & 4 & 4,9 & 5,0 & 19,2 & $-0,66$ & 0,20 & 3 \\
\hline Confiabilidade ao projeto & 5,6 & 6,0 & 12,0 & $-1,46$ & 1,39 & 3 & 4,6 & 5,0 & 32,4 & $-0,54$ & $-1,27$ & \\
\hline Desempenho do equipamento & 5,6 & 6,0 & 12,0 & $-1,46$ & 1,39 & 2 & 5,6 & 6,0 & 16,8 & $-2,41$ & 6,06 & 1 \\
\hline Confiabilidade do equipamento & 5,7 & 6,0 & 15,7 & $-2,95$ & 8,89 & 1 & 4,3 & 5,0 & 34,9 & $-0,57$ & $-1,27$ & \\
\hline Flexibilidade de alteração técnica & 4,3 & 4,0 & 30,3 & 0,06 & $-0,66$ & & 3,8 & 4,0 & 43,5 & $-0,77$ & $-0,39$ & \\
\hline Média geral e $\alpha$-Cronback & 4,9 & \multicolumn{2}{|c|}{$\alpha_{\mathrm{T}}=0,866$} & \multicolumn{3}{|c|}{$\alpha_{4}=0,815$} & 4,4 & \multicolumn{2}{|c|}{$\alpha_{T}=0,043$} & \multicolumn{3}{|c|}{$\alpha_{4}=0,698$} \\
\hline
\end{tabular}

Fonte: Dados da pesquisa

Tomando individualmente o elemento destacado no início dessa análise e que revelou considerável desalinhamento nesse elo, há uma indicação de que é necessário um 
relacionamento mais próximo com o cliente (empresa foco) para que o fornecedor possa promover a imagem de ter um produto confiável. Significa que essa aproximação pode representar uma oportunidade para que o fornecedor melhore sua posição competitiva.

Da mesma forma que no elo a jusante, a distribuição de dados mostrou-se ser não normal. O lado da empresa foco da área de compras (3) apresentou coeficiente de variação de menor amplitude, maior pontuação de escala somada, melhor consistência interna, incluindo as quatro primeiras prioridades.

\subsection{Comparação Indutiva dos Diferentes Métodos de Pesquisa}

Realizada a análise exploratória dos dados, buscou-se indagar se eles eram estatisticamente significantes. Ainda mais, verificar se a coleta de dados por métodos diferentes apresentavam os mesmos resultados. Assim, para obter estas respostas, foram aplicados testes não paramétricos de Mann-Whitney, Kolmogorov-Sminov e Qui_Quadrado. Os dados obtidos, tanto na primeira quanto na segunda fase, foram submetidos aos testes de hipóteses em nível de significância $(\alpha \leq 0,05)$. Os resultados, representados pelas colunas ( 1 2) e $(3-4)$, são mostrados na Tabela 6 . Observa-se que no elo a jusante, considerando o cliente (1) e área de vendas da empresa foco (2), as prioridades flexibilidade de alteração contratual e flexibilidade de alteração técnica são significantes $(\alpha \leq 0,05)$ quando se aplica os testes de Mann-Whitney, Kolmogorov-Sminrnov e do Qui-Quadrado, denotando coerência nos resultados obtidos. Todavia, vale lembrar que na análise da escala somada, esses critérios foram considerados pouco importantes, o que reforça que essas não são prioridades nem a montante nem a jusante na cadeia de suprimentos estudada.

Tabela 6: Testes de significância $(\alpha \leq 0,05)$

\begin{tabular}{|c|c|c|c|c|c|c|c|}
\hline \multirow[t]{2}{*}{ PRIORIDADES } & \multicolumn{3}{|c|}{$\begin{array}{l}\text { Mann- } \\
\text { Whitney }\end{array}$} & \multicolumn{2}{|c|}{$\begin{array}{l}\text { Kolmogorov- } \\
\text { Smirnov }\end{array}$} & \multicolumn{2}{|c|}{$\chi^{2}($ Qui } \\
\hline & & $1-2$ & $3-4$ & $1-2$ & $3-4$ & $1-2$ & $3-4$ \\
\hline \multirow{2}{*}{ A1 Flexibilidade de } & $\mathrm{Oi}$ & 0,447 & 0,891 & & 0,998 & 0,399 & 0,572 \\
\hline & Ec & 0,087 & 0,190 & 0,473 & 0,910 & 0,487 & 0,630 \\
\hline \multirow{2}{*}{ A2 Preço (equipamento } & $\mathrm{Oi}$ & 0,628 & 0,053 & 0,977 & 0,229 & 0,260 & 0,151 \\
\hline & Ec & 0,769 & 0,059 & 0,591 & 0,292 & 0,102 & 0,123 \\
\hline \multirow{2}{*}{$\begin{array}{l}\text { A3 Velocidade da data } \\
\text { de entrega }\end{array}$} & Oi & 0,163 & 0,053 & 0,387 & 0,393 & 0,104 & 0,274 \\
\hline & Ec & 0,137 & 0,976 & 0,073 & 0,979 & 0,021 & 0,178 \\
\hline \multirow{2}{*}{ A4 $\begin{array}{l}\text { Confiabilidade da } \\
\text { data de entreoa }\end{array}$} & Oi & 0,396 & 0,270 & & 0,864 & 0,375 & 0,565 \\
\hline & Ec & 0,938 & 0,316 & 1,000 & 0,928 & 0,741 & 0,288 \\
\hline \multirow{2}{*}{ 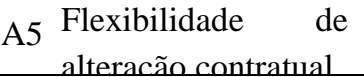 } & Oi & & 0,761 & & 0,925 & & 0,233 \\
\hline & Ec & 0,001 & 0,566 & 0,011 & 1,000 & 0,040 & 0,744 \\
\hline \multirow{2}{*}{ E1 Confiabilidade às } & Oi & 0,409 & 0,837 & & 0,995 & 0,677 & 0,630 \\
\hline & Ec & 0,745 & 0,211 & 1,000 & 0,928 & 0,911 & 0,514 \\
\hline \multirow{2}{*}{ E2 Confiabilidade ao } & Oi & 0,052 & 0,440 & 0,167 & 0,928 & 0,096 & 0,434 \\
\hline & Ec & 0,722 & 0,169 & 1,000 & 0,408 & 0,914 & 0,288 \\
\hline \multirow{2}{*}{ E3 Desempenho do } & Oi & 0,221 & 0,183 & 0,389 & 0,318 & 0,229 & 0,071 \\
\hline & Ec & 0,914 & 0,880 & 1,000 & 1,000 & 0,508 & 0,540 \\
\hline \multirow{2}{*}{ E4 Confiabilidade do } & Oi & 0,418 & 0,158 & 0,726 & 0,766 & 0,236 & $\mathbf{0 , 0 2 3}$ \\
\hline & Ec & 0,545 & 0,006 & 0,803 & $\mathbf{0 , 0 1 5}$ & 0,143 & 0,016 \\
\hline \multirow{2}{*}{ E5 Flexibilidade de } & Oi & 0,171 & 0,429 & 0,258 & & 0,124 & 0,390 \\
\hline & Ec & 0,003 & 0,695 & $\mathbf{0 , 0 1 9}$ & 0,991 & 0,010 & 0,217 \\
\hline
\end{tabular}

Oi - Coletas por ordem de importância - Amostras: $\mathrm{n}_{1}=51, \mathrm{n}_{2}=10, \mathrm{n}_{3}=10, \mathrm{n}_{4}=42$

Ec - Coletas pela escala de concordância - Amostras: $\mathrm{n}_{1}=26, \mathrm{n}_{2}=12, \mathrm{n}_{3}=12, \mathrm{n}_{4}=11$ 


\section{ALINHAMENTO ESTRATÉGICO NA CADEIA DE SUPRIMENTOS: UMA ANÁLISE DE EMPRESAS DO SETOR DE BENS DE CAPITAL A PARTIR DE SUAS PRIORIDADES COMPETITIVAS}

Fonte: Dados da Pesquisa

Observa-se que o único teste não-paramétrico realizado para os dados coletados por ordem de importância (Oi) que apresentou significância $(\alpha \leq 0,05)$ ocorreu quando se aplicou o teste de Qui-Quadrado para o critério E4 (confiabilidade do equipamento) no elo entre o fabricante e o fornecedor. Levando-se em conta ainda que os outros testes não-paramétricos apresentaram resultados significantes para a coleta de dados realizada por meio da escala de concordância, este se torna um critério que demanda especial atenção no relacionamento entre fornecedor e fabricante, devendo estar alinhado como prioridade estratégica para este elo da cadeia.

Outro ponto a destacar é o fato do critério preço (A2) não ter apresentado significância em nenhum dos testes não-paramétricos para ambos os métodos de coleta de dados. Tal observação chama a atenção tendo em vista que o critério preço apareceu claramente como principal prioridade para cliente e fabricante ao se utilizar a ordem de importância.

Por fim, notou-se que a velocidade de entrega (A3) mostrou-se significante para o teste Qui-Quadrado na relação entre cliente e fabricante pela escala de concordância. Esse critério ficou em uma posição intermediária na análise das escalas somadas, tanto pelas respostas dos clientes quanto pelas respostas dos fabricantes. Sugere-se que a velocidade de entrega, apesar de não ser prioritária frente a confiabilidade da entrega, não pode ser desprezada a fim de não colocar em risco a imagem de pontualidade que o fabricante veicula e que é importante para o cliente. Vale lembrar ainda, que Brown et al. (2006) alertam para o fato das prioridades competitivas poderem alterar ao longo do tempo, e também Slack et al. (2002) que destacam a importância de se considerar a inter-relação dos objetivos de desempenho na definição e operacionalização das prioridades competitivas.

\section{CONCLUSÕES E SUGESTÕES PARA PROSSEGUIMENTO}

O objetivo central desse artigo foi verificar se as empresas do setor de bens de capital mecânico possuem prioridades competitivas que se alinhem às suas estratégias e às estratégias de seus parceiros ao longo da cadeia de suprimentos.

Foi possível verificar que as empresas pesquisadas estão bastante preocupadas com seu processo produtivo, dando ênfase ao desempenho do equipamento adquirido, de forma que possa cumprir o que fora prometido aos clientes. Essa é uma preocupação que se verificou ao longo do trecho da cadeia estudada, ou seja, a montante e a jusante da empresa foco. Constatou-se ainda que, no elo a montante do fabricante, a flexibilidade técnica e comercial, assim como o preço não são prioridades para a definição dos critérios competitivos.

De uma maneira geral, os resultados encontrados mostram clara orientação das empresas em serem competitivas, seja pela prática de preços competitivos ou pela imagem de confiança que pretendem passar aos seus clientes. Todavia, alguns descompassos encontrados em alguns critérios competitivos apontaram para a necessidade de maior aproximação entre as empresas e estabelecimento de um relacionamento mais cooperativo para o alinhamento estratégico. Os resultados da pesquisa confirmam a presença de desafios relevantes em termos de alinhamento estratégico, especialmente a montante da cadeia onde se identifica um número 
menor de relacionamentos gerenciados do que a jusante. Dessa forma, fica evidente que a prática da integração ao longo da cadeia de suprimentos das empresas do setor de bens de capital ainda apresenta potencial de crescimento, para que haja alinhamento estratégico entre fornecedor, fabricante e cliente, podendo gerar melhor utilização de recursos, velocidade e qualidade (COX et al., 2004; CROXTON et al., 2001).

Quando se utilizou o método da escala de ordem de importância e aplicação da estatística descritiva, verificou-se que o preço se mostrou como a principal prioridade competitiva alinhada entre clientes e a área de vendas da empresa foco. Todavia este resultado não se confirmou quando o método foi alterado para o da escala de concordância. Quanto à significância estatística das prioridades competitivas, pela aplicação do método da escala da concordância verificou-se que as prioridades flexibilidade de alteração contratual e flexibilidade de alteração técnica são significantes na relação entre clientes e a área de vendas da empresa foco, denotando que elas não compartilham das mesmas prioridades.

Ao se comparar os dois métodos de coletas de dados utilizados na pesquisa empírica, apurou-se resultados distintos, revelando a necessidade de se ter a preocupação em apontar e considerar o método a ser utilizado para a interpretação do fenômeno estudado. Assim, destaca-se a importância em se ter o cuidado de explicar os procedimentos metodológicos utilizados para que os artigos com resultados empíricos tragam real contribuição para a construção do arcabouço teórico e sejam referências úteis aos gestores da área de operações e supply chain.

Para prosseguimento da pesquisa, sugere-se concentrar no método de coleta de dados por meio da escala de classificação não comparativo (ou escala de concordância) e periodicamente, coleta de dados mantendo a amostra de empresas fixa, tornando-a de concepção transversal para longitudinal. Desse modo, a análise do alinhamento estratégico empírico frente às teorias, se tornará mais consistente, uma vez que os resultados poderão incorporar a dinâmica dos processos de mudanças ocorridos no intervalo de abrangência do estudo.

\section{REFERÊNCIAS}

BALLOU, Ronald H. The evolution and future of logistics and supply chain management. Produção. v.16, n.3, p.375, Set/Dez 2006.

BARNEY, Jay B. Looking inside for competitive advantage. Academy of Management Executive. V.9, n.4, p.49-61. Ada: novembro, 1995.

BNDES (Banco Nacional de Desenvolvimento Econômico e Social). Disponível em <http://www.bndes.gov.br>. Acessado em fevereiro de 2010.

BROWN, Steve; LAMMING, Richard; BESSANT, John; JONES, Peter. Administração da Produção e Operações. Rio de Janeiro: Editora Campus / Elsevier, 2006. 
ALINHAMENTO ESTRATÉGICO NA CADEIA DE SUPRIMENTOS: UMA ANÁLISE DE EMPRESAS DO SETOR DE BENS DE CAPITAL A PARTIR DE SUAS PRIORIDADES COMPETITIVAS

CHASE, Richard B.; AQUILANO, Nicholas J.; JACOBS, F. Robert. Administración de producción y operaciones: manufactura y servicios. Madrid: Mosby/Doyma Libros, 2001.

CHOPRA, Sunil; MEINDL, Peter. Gerenciamento da Cadeia de Suprimentos: estratégia, planejamento e operação. São Paulo: Prentice Hall, 2003.

COX, A.; LONSDALE, C.; SANDERSON, J. e WATSON, G. Business Relationships for Competitive Advantage: Managing Alignment and Misalignment in Buyer and Supplier Transactions. Palgrave MacMillan. Nova York, US. 2004.

CROXTON, K. L.; DASTUGUE, S. J.; LAMBERT, D. M. e ROGERS, D. The supply chain management processes. International Journal of Logistics Management. Vol.12, $\mathrm{n}^{\mathrm{o}} 2$; pg. $13,2001$.

DE MEYER, Arnoud; NAKANE, Jinichiro; MILLER, Jaffrey G; FERDOW, Kasra. Flexibility: the next competitive battle - the manufacturing futures survey. Strategic Management Journal, v.10, p.135-144, 1989.

DROGE, Cornelia; VICKERY, Shawnee; MARKLAND, Robert E. Sources and outcomes of competitive advantage: An Exploratory Study in the Furniture Industry. Decision Sciense. v.25, n.5,6; p. 669. Atlanta: Sep-Dec 1994.

FLYNN, Barbara B, FLYNN, E James. An exploratory study of the nature of cumulative capabilities. Journal of Operations Management. v.22, n.5, p.439-457. Columbia: Oct, 2004.

HAIR, Joseph F. Fundamentos de métodos de pesquisa em administração. Porto Alegre: Bookman, 2005.

HAYES, R.W., PISANO, G. P. Manufacturing Strategy: at the intersection of two paradigm shifts. Production \& Operations Management, v.5, n.1, p.25-41, Spring 1996.

HEIZER, Jay; RENDER, Barry. Administração de Operações, Bens e Serviços. Rio de Janeiro: Edtiora LTC, 2001.

HILL, T. Manufacturing Strategy: Text and Cases. London: Mcmillian Business, 1993.

IBGE. Instituto Brasileiro de Geografia e Estatísticas. <www.ibge.gov.br>. Acessado em 12 janeiro de 2008 .

IMAM. Inovação e Melhoramento na Administração Moderna. <http://www.imam.com.br>. Acessado em 31 de janeiro de 2009.

LAMBERT, Douglas M; COOPER, Martha C. Issues in Supply Chain Management. Industrial Marketing Management. v.29, n.1, p.65. New York: Jan, 2000. 
LAU, R. S. M. Competitive factors and their relative importance in the US electronics and computer industries. International Journal of Operations \& Production Management. v.22, n.1, p,125-135. 2002.

MALONI, M. e BENTON, B. C. Power Influences in the Supply Chain. Journal of Business Logistics. Vol. 21, nº 1, pp. 49-73, 2000.

PAIVA, Ely Laureano; CARVALHO Jr, José Mário; FENSTERSEIFER, Jaime Evaldo. Estratégia de Produção e de Operações. Porto Alegre: Bookman, 2004.

SEBRAE (Serviço Brasileiro de Apoio às Micro e Pequenas Empresas). www.sebrae.com.br. Acessado em fevereiro de 2010.

SLACK, Nigel. Vantagem Competitiva em Manufatura. São Paulo: Editora Atlas, 1993.

SLACK, Nigel. Operations strategy: will it ever realize its potential?. Gestão \& Produção. v.12, n.3, p.323-332. Dez, 2005.

SLACK, Nigel; CHAMBERS, Stuart; JOHNSTON, Robert. Administração da produção. São Paulo: Atlas, 2002.

TAN, Keah Choon. A framework of supply chain management literature. European Journal of Purchasing \& Supply Management. v.7, p.39. April, 2000.

VOKURKA, R. J.; ZANK, G. M.; LUND, C. M. Improving competitiveness through supply chain management: A cumulative improvement approach. Competitiveness Review, Vol. 12, $\mathrm{n}^{\mathrm{o}} 1$, pp. 14, 2002.

WERNERFELT, B. A Resource-based View of The Firm. Strategic Management Journal. Vol. 5, 2, 1984. 